$\xi=-$

\title{
Asymptotic stability of nonlinear integrodifferential equation
}

\author{
Jackreece P. C. ${ }^{1 *}$, Aniaku S. E. ${ }^{2}$ \\ ${ }^{1}$ Department of Mathematics and Statistics, University of Port Harcourt, Nigeria \\ ${ }^{2}$ Department of Mathematics, University of Nigeria, Nsukka, Nigeria \\ *Corresponding author E-mail: prebo.jackreece@uniport.edu.ng
}

\begin{abstract}
The investigation of stability for nonlinear dynamical system often related to the construction of Lyapunov functionals. We employ Lyapunov functionals to the system of nonlinear Volterra integro-differential equations of the form $y^{\prime}(t)=B(t) g(y(t))+\int_{0}^{t} G(t, s, y(s))$ and obtain conditions for the asymptotic stability of the zero solution. Also, we give examples to illustrate the obtained results.
\end{abstract}

Keywords: Asymptotic Stability; Lyapunov Functional; Integro-Differential Equations.

\section{Introduction}

Nonlinear integro-differential equations have been widely studied by researchers due to its wide applications in biology, ecology, medicine, physics, engineering and other scientific areas, (see [3], [5], [20] and reference therein).

In recent years, qualitative properties of solutions such as stability, instability, boundedness, existence etc. of Volterra integrodifferential equations have been extensively investigated by different authors, [1], [3], [4], [10], [11], [16].

An important tool employed to discuss the qualitative properties of solutions of dynamical systems is the Lyapunov method. Alexander Lyapunov was the first to define the notion of stable system [12]. The stability theorem for motion studied by A.M Lyapunov has proven to be highly useful and applicable in the field of science and engineering. Over the years, Lyapunov method for the stability of integro-differential equation have been proposed by different researcher ([8], [13], [14], [15], [16], [17], [19]).

In particular, [7] developed a Lyapunov theory and used nonincreasing or strictly decreasing Lyapunov functionals along solutions that primarily seems to apply to Volterra integro-differential equations. Theoretically, this method is very appealing and there are numerous applications in which it is used in nature. [15] worked on the stability of the zero-state solution of impulsive function differential equation. They applied the LyapunovRazumikhin method and Piecewise continuous function to check the behavioral solution of equation. [19] established stability of systems of Volterra integro-differential equation. They used a known form of Lyapunov functional to establish the stability condition for the system. Also, [13] establishes the stability of the solutions of a class of integro-differential equations of Volterra type whose nonlinear term is assumed to be holomorphic function of variables and possible some integral form in a small neighborhood of zero. Stability in Lyapunov's sense of single zero root and of pair of pure imaginary roots for the unperturbed equation is analyzed by relying on functional in integral form represented by Fréchet series. [8] worked on construction of Lyapunov functionals to check and investigate the stability for hereditary system. [18] studied certain nonlinear Volterra integro differential equa- tions with delay. He established stability and boundedness condition of the solution by defining a suitable Lyapunov functional used to prove the result.

\section{Preliminaries}

Our aim in this paper is to use a suitable Lyapunov functional and determine necessary and sufficient condition for the stability of the zero solution of the nonlinear integro - differential equation of Volterra type defined by

$y^{\prime}(t)=B(t) g(y(t))+\int_{0}^{t} G(t, s, y(s))$

Where $y \in R$, the functions $\mathrm{G}$ is continuous in $(\mathrm{t}, \mathrm{s}, \mathrm{y})$ for $0 \leq$ $\mathrm{s} \leq \mathrm{t}<\infty \quad, \quad \mathrm{B}(\mathrm{t}) \quad$ continuous for $0 \leq \mathrm{t}<\infty$, $\mathrm{g}(\mathrm{y}(\mathrm{t}))$ is continuous on $(-\infty, \infty)$ and

$\int_{0}^{t} G(t, s, y(s)) d s<\infty, \int_{0}^{t} t G(t, s, y(s)) d s<\infty$

For any $t_{0} \geq 0$ and initial function $\varphi \in\left[t_{0}, t\right]$, let $y(t)=y\left(t, t_{0}, \varphi\right)$ denote the solution of eq. (2.1) on $\left[t_{0}, t\right]$ such that $y(t)=\varphi(t)$. Let $C\left(\left[t_{0}, t_{i}\right]\right)$ and $C\left(\left[t_{0}, \infty\right]\right)$ denote the continuous real valued functions on $\left[t_{0}, t_{1}\right]$ and $\left[t_{0}, \infty\right]$ respectively. For $\phi \in C\left[t_{0}, 0\right]$, $|\phi|=\sup \left\{|y(t)|: 0 \leq t \leq t_{0}\right\}$.

Definition 2.1: The zero solution of eq. (2.1) is stable if for each $\varepsilon>0$ and each $t_{0} \geq 0$, there exist $\delta(\varepsilon)>0$ such that $\|\varphi\|_{t_{0}}<\delta(\varepsilon)$ which implies that $\left\|y\left(t, t_{0}\right)\right\|<\varepsilon$ for $t \geq 0$ where $y(t, \varphi)$ is a solution of eq. (2.1) which is defined for $t \geq t_{0}$.

Definition 2.2: The zero solution of eq. (2.1) is uniformly stable if for each $\varepsilon>0$ there exist $\delta=\delta(\varepsilon)>0$ such that $\varphi \in\left[0, t_{0}\right]$ with $\|\varphi\|<\delta$ (any $t_{0} \geq 0$ implies that $\|y(t, \varphi)\|<\varepsilon$ for all $t \geq t_{0}$. 
Definition 2.3: The function $f(t, y): R^{+} \times X \rightarrow X$ is called Lipchitz in $y$ if $\forall n>0, \exists L \geq 0$ such that, $\| f\left(t, y_{1}\right)-f\left(t, y_{2} \| \leq\right.$ $\left\llcorner\left\|y_{1}-y_{2}\right\|\right.$, for all $y_{1}, y_{2} \in B_{n}, t \geq 0$ where $\llcorner$ is called Lipschitz constant and $B_{n}$ is a close ball with radius $n$.

Definition 2.4: The zero solution of eq. (2.1) is said to be asymptotically stable if it is stable and there is a number $\delta>0$ such that any solution $y(t)$ with $\|\varphi\|<\delta$ satisfies $\lim _{t \rightarrow \infty}\|y(t)\|=0$.

Definition 2.5: Suppose $\phi \in R^{n}$ for each solution $y(t, \phi)$ and if $\exists B\left(y_{0}\right)$ such that $\left|y\left(t, y_{0}\right)\right| \leq B\left(y_{0}\right)$ for $t \geq 0$. Then the solution of eq. (2.1) is bounded.

The following theorem is essential for stability result and is a basic tool for our results.

Theorem 2.1: [9]. If there exists a functional $V(t, \varphi()$.$) , defined$ whenever $t \geq t_{0} \geq 0$ and $\varphi \in C\left([0, t], R^{n}\right)$ such that

i) $\quad V(t, 0) \equiv o, V$ is continuous in t and locally Lipschitz in $\phi$

ii) $\quad V(t, \varphi(t)) \geq W(|\varphi(t)|), W:[0, \infty) \rightarrow[0, \infty)$ is a continuous function with $W(0)=0 W(r)>0$, ifr $>0$ and W is strictly increasing (positive definiteness), and

iii) $V^{\prime}(t, \varphi()) \leq$.0 then the zero solution of eq. (2.1) is stable and

iv) $V(t, \varphi())=.V(t, \varphi(s)): 0 \leq s \leq t$

Is called a Lyapunov function of eq. (2.1)

\section{Main result}

Theorem 3.1: If $\mathrm{B}(\mathrm{t})<0, \mathrm{G}(\mathrm{t}, \mathrm{s}, \mathrm{y}(\mathrm{s}))>0$ and

$$
B(t) g(y(t))+\int_{0}^{t} G(t, s, y(s)) d s \neq 0
$$

Then the statements below are equivalent.

i) The solution of eq. (2.1) tends to zero.

ii) $\mathrm{B}(\mathrm{t}) \mathrm{g}(\mathrm{y}(\mathrm{t}))+\int_{0}^{\mathrm{t}} \mathrm{G}(\mathrm{t}, \mathrm{s}, \mathrm{y}(\mathrm{s})) \mathrm{ds}<-\xi, \xi>0$

iii) Every solution of eq. (2.1) is a Lebesgue integrable function with respect to the vector space $\mathrm{R}^{\mathrm{n}}$. For the proof see [11].

Theorem 3.2: (Strong Condition for Stability) If

$B(t) g(y(t))+\int_{0}^{\infty} G(t, s, y(s)) d t<0$

Then the zero solution of eq. (2.1) is uniformly asymptotically stable

Proof

We choose a Lyapunov functional

$V(t, s, g(y(t)))=|y(t)|$

$+\int_{0}^{t} \int_{0}^{\infty}|G(\beta, s, y(s))| d \beta|g(y(s))| d s$

Then $y \neq 0$, where $y(t)$ is assumed solution of eq. 2.1. We have

$$
\begin{aligned}
& V^{\prime}(t, s, g(y(t))) \leq B(t)|g(y(t))|+\int_{0}^{t}|G(t, s, y(s))||g(y(s))| d s \\
& +\int_{0}^{\infty}|G(\beta, s, y(s))| d \beta|g(y(t))|-\int_{0}^{t}|G(t, s, y(s))||g(y(s))| d s \\
& =B(t)|g(y(t))|+\int_{0}^{\infty}|G(\beta, s, y(s))| d \beta|g(y(t))| \\
& =|g(y(t))|\left[B(t)+\int_{0}^{\infty}|G(\beta, s, y(s))| d \beta\right] V^{\prime}(t, s, g(y(t))) \\
& =-|g(y(t))| \xi
\end{aligned}
$$

For some positive constant $\xi>0$ and $g(y(t))$ is Lebesgue integrable, hence the equilibrium solution is uniformly asymptotically stable.

Theorem 3.3: (Weak condition for stability) A weak condition for stability of eq. 2.1 is given below

$B(t) g(y(t))+\left|\int_{0}^{\infty} G(t, s, y(s)) d t\right|>0$

Proof

Choosing the usual Lyapunov functional

$$
\begin{aligned}
& V(t, s, g(y(t)))= \\
& |y(t)|\left|\int_{0}^{t} \int_{0}^{\infty} G(\beta, s, y(s)) d \beta g(y(s)) d s\right|
\end{aligned}
$$

Then for all $y \neq 0$, assuming $y(t)$ is a solution of eq. (3.4), differentiating eq. (3.5) along the solution of eq. (3.5), then we have

$$
\begin{aligned}
& V^{\prime}(t, s, g(y(t))) \leq|B(t) g(t(t))|+\left|\int_{0}^{t} G(t, s, y(s)) d s\right| \\
& +\left|\int_{0}^{\infty} G(\beta, s, y,(s)) d \beta g(y(t))\right| \\
& -\left|\int_{0}^{t} G(t, s, y(s)) g(y(s)) d s\right| \leq|B(t) g(y(t))| \\
& +\left|\int_{0}^{\infty} G(\beta, s, y(s)) d \beta g(y(t))\right| \\
& =|g(y(t))|\left[\left[B(t)+\int_{0}^{\infty} G(\beta, s, y(s)) d \beta\right] \mid\right. \\
& \quad V^{\prime}(t, s, g(y(t)))=|g(y(t))-\xi|
\end{aligned}
$$

Hence the solution of eq. (2.1.) is unstable under theorem 3.3 even if $G(t, s, y(s))$ is Lebesgue integrable and bounded.

Theorem 3.4: (Necessary and sufficient condition) Suppose

i) $\varphi: R_{+} \rightarrow R$ is continuous and $G(t, s, y)$ is continuous for $0 \leq s \leq t \leq \infty$ and satisfy the Lipschitz condition.

$$
\begin{aligned}
& \left\|B(t) g\left(y_{1}(s)\right)-B(t) g\left(y_{2}(s)\right)\right\| \\
& +\left\|\int_{0}^{t} G\left(t, s, y_{1}\right) d s-\int_{0}^{t} G\left(t, s, y_{2}\right) d s\right\| \\
& \quad \geq L_{1}\left\|y_{1}-y_{2}\right\|+\varphi L_{2}\left\|y_{1}-y_{2}\right\|
\end{aligned}
$$

For all

$\left\|t-t_{0}\right\| \leq \alpha,\left\|y_{1}\right\|<\infty,\left\|y_{2}\right\|<\infty$ and $\varphi>0$. Then solution of eq. (2.1) is unique.

ii) If the integral $\int_{0}^{t}|G(\beta, s, y(s))| d s$ is defined and continuous for $0 \leq s \leq t \leq \infty$ and given positive number $\xi$ such that

$$
2 B(t)+\int_{0}^{t} G(t, s, y) d s+\int_{0}^{\infty} G(\beta, s, y) d \beta \leq-\xi
$$

Then the zero solution is stable if and only if $G(t)<0$. (for proof, see [11]).

\section{Theorem 3.5: If}

$$
\begin{aligned}
& 2 B(t)+\int_{0}^{t} \mid G(t, s, y(s)) d s \\
& +\int_{t}^{\infty}|G(\beta, t, y(s))| d \beta \leq-\xi \\
& \text { and } G(t)<0
\end{aligned}
$$

Holds and bounded, then the zero solution of eq. (2.1) is asymptotically stable.

Proof 
We have proved from theorem (3.4) that the derivative of the Lyapunov functional is less than or equal to zero i.e.

$V^{\prime}(t, s, g(y(t))) \leq-\xi g\left(y^{2}(s)\right)$ that $g\left(y^{2}(s)\right)$ is Lebesgue integrable and is bounded on $\mathrm{R}^{+}$, hence $g(y(s)) \rightarrow 0$ as $t \rightarrow \infty$.

We therefore conclude that the zero solution is asymptotically stable.

We now need to obtain the sufficient conditions for the asymptotic stability of eq. (2.1) in which $G(t)$ is not negative and $G(t, s, y(s))$ need not be integrable on $\mathrm{R}_{+} \times \mathrm{R}_{+}$., here eq. (2.1) is equivalent to

$$
\begin{aligned}
& V^{\prime}=g(y(t)) V(t) \\
& +\int_{0}^{t} A(t, s, y(s)) V(s) d s+B(t, s)
\end{aligned}
$$

And

$$
\begin{aligned}
& A(t, s, y(t))=G(t, s, y(t))+\frac{\partial m}{\partial s}(t, s, y(t)) B(s, y(s)) \\
& +\int_{0}^{t} m(\beta, s, y(s)) d \beta
\end{aligned}
$$

\section{Where}

$B(t, s)=m(t, s, 0) V(0), m(t, s, y(s))$ Being a continuously differentiable function for

$0 \leq s \leq t \leq \infty$ and $g(y(t))=B(t)-m(t, t, t)$.

Also, if we assume that there exist a $A(t, s, y(s))$ which is continuously differentiable for $0 \leq s \leq t \leq \infty$ such that

a) $|m(t, s, 0)| \rightarrow 0$ as $t \rightarrow \infty$ and $\int_{0}^{t}|m(t, s, 0)| d t<\infty$

b) $\int_{0}^{\prime}|m(\beta, s, y(s))| d \beta$ is define and finite for all $\geq 0$.

Then, if $\exists \xi>0$ such that

$$
\begin{aligned}
& \int_{0}^{t}|A(t, s, y(s))| d s+\int_{0}^{\infty} A(\beta, s, y(s)) d \beta \\
& +|m(t, s, 0)|+2 g(y(t)) \leq-\xi
\end{aligned}
$$

If $g(y(s))<0$ and bounded, then the zero solution of eq. (2.1) is asymptotically stable.

Theorem 3.6: Assume that condition (a) and holds with

$$
\begin{aligned}
& m(t, s, y(s))=m(t-s, t-y(s)) \\
& \text { and } A(t, s, y(s))=A(t-s, t-y(s))
\end{aligned}
$$

Holds if

$B(t) g(y(s))+\int_{0}^{\infty} A(t, s, y(s)) d s<0$, then the zero solution of eq. (2.1) is uniformly asymptotically stable.

We shall consider the example below to show the validity of the theorem above.

Example 1: Consider the integro - differential equation.

$$
y^{\prime}=-10 g(t)-\int_{0}^{t} 24 e^{2^{(t-s)}} d s
$$

Then,

$g(y(t))=B(t)-m(t, t, t)=-10-(-6)=-10+6=-4<0$

Hence the zero solution of eq. (2.1) is asymptotically stable. Example 2: Consider the scalar equation

$$
\begin{gathered}
y^{\prime}=\frac{2}{12+t} g(t) \\
-\int_{0}^{t} \frac{12 e^{-10(t-s)}(4+s)(3+s) \cos (s)}{(3+t)^{2}}
\end{gathered}
$$

Comparing with eq. (2.1), we have that

$B(t)=\frac{2}{12+t}, G(t, s, y(s))=-\frac{12 e^{-10(t-s)}(4+s)(3+s) \cos (s)}{(3+t)^{2}}$,

Select, $m(t, s, y(s))=\frac{2 e^{-2(t-s)}(4+s)^{2} \cos (s)}{(3+t)^{2}}$

Then,

$$
\begin{aligned}
& G(y(t))=B(t)-m(t, t, t) \\
& =\frac{2}{12+t}-\frac{2(4+s)^{2} \cos (t)}{(3+t)^{2}}<0 \quad \forall t \geq 0
\end{aligned}
$$

Also,

$$
\begin{aligned}
& A(t, s, y(s))=G(t, s, y(s)) \\
& +\frac{2 m(t, s, y(s))}{2 s}+B(t) \cdot m(t, s, y(s)) \\
& +\int_{0}^{t} m(t, s, y(s)) \cdot G(t, s, y(s)) d t \\
& A(t, s, y(s))=-\frac{\sin t}{\left(3+t^{2}\right)}-\frac{4 \cos t}{(3+t)^{2}} \\
& \quad+\frac{4 \cos t}{(12+t)(3+t)^{2}} \\
& \Rightarrow G(t, s, y(s))+B(t) \cdot m(t, s, y(s)) \\
& +\int_{0}^{t} 2|A(t, s, y(s))| d s+|m(t, 0, y(0))|-4|y(t)| \\
& \Rightarrow \frac{-12 \sin (t)}{(3+t)^{2}}+\frac{2\left(e(-4)^{-2 t}\right)}{(3+t)^{2}} \\
& -\frac{8}{12+t} 8\left|\frac{\cos (t)}{(3+t)^{2}}\right| \leq \frac{-4}{9}
\end{aligned}
$$

Thus,

$$
\xi=\frac{4}{9}
$$

Having satisfied the asymptotic stability condition, hence the zero solution is asymptotically stable.

Comparing with equation (4.1), we have here that

$\mathrm{B}(\mathrm{t})=-10, G(t, s, y(s))=-24 e^{2}(t-s)$

Choose

$m(t, s, y(s))=-6 e^{(t-s)}, m(t, t, t)=-6 e^{0}=-6$ 


\section{References}

[1] M. Adivar and Y. N. Raffoul., Inequalities and exponential stability in finite delay Volterra integro-differential equations, Rend. Circ $\begin{array}{llll}\text { Mat. } & \text { Palermo (2) 61, 321-330. }\end{array}$ https://doi.org/10.1007/s12215-012-0092-4.

[2] L. C. Becker, Uniform continuous $L^{1}-$ solution of Volterra equations and global asymptotic stability, Cubo 11, (2009): 1-24.

[3] T. A. Burton, Volterra integral and differential equations Second edition, Mathematics in Science and Engineering, 202, Elsevier B. V. Amsterdam, 2005.

[4] T. A. Burton, Stability theory for Volterra equations, J. Differential equations 32, 1, (1979): 101-118.

[5] T. A. Burton and W. E. Mahfoud, Stability criteria for Volterra equations, Trans. Amer. Soc. 279, 1, (1983):143-174.

[6] T. A. Burton and J. R. Haddock, Qualitative properties of solutions of integral equations, Nonlinear Anal. 71, No. 11, (2009):57125723. https://doi.org/10.1016/j.na.2009.04.047.

[7] T. A. Burton, Q. C. Huang and W. E. Mahfoud, Rate of decay of solutions of Volterra equations, Nonlinear Anal. 9(7), (1985): 651663. https://doi.org/10.1016/0362-546X(85)90011-2.

[8] T. Caraballo, J. Real. and L. Shaikhet, Method of Lyapunov functional construction in stability of delay evolution equation. J. Math. $\begin{array}{lll}\text { Anal. } & 334 . & \text { (2007):1130-1145 }\end{array}$ https://doi.org/10.1016/j.jmaa.2007.01.038.

[9] R. Driver, Existence and stability of solution of a delay-differential system, Arch. Rat. Mech. Anal., 10, (1962):401-426. https://doi.org/10.1007/BF00281203.

[10] P. Eloe , M. Islam and B. Zhang, Uniform asymptotic stability of linear Volterra integro-differential equations with application to delay systems, Dynam. Systems Appl. 9, No. 3, (2000)331-344.

[11] P. C. Jackreece and S. Aniaku, Stability Results of Nonlinear Integro-differential Equations. Mathematical Theory and Modeling, 8(1), (2018): 27-33.

[12] K. Magnus, Development of the stability concept in mechanics. Naturwissenshaften. 46, (1959):590-595 https://doi.org/10.1007/BF00684205.

[13] V. S. Sergeev, Stability of solutions of Voterra integro-differential equations. Mathematical and computer modeling. 45, (2007):13761394. https://doi.org/10.1016/j.mcm.2006.09.023.

[14] I. M. Stamova and G. T. Stamov, Analysis of differential equation with maximum, Math. Slovaca, 63, (6)(2013): 1291-1302. https://doi.org/10.2478/s12175-013-0171-9.

[15] I. M. Stamova, and G. T. Stamov, Lyapunov-Razumikhim method for impulsive functional differential equations and application to population dynamics, J. compt. Appl. Math. 130, (2001):163-171

[16] C. Tunç and S. Abid, Journal of the Egyptian Mathematical Society A remark on the stability and boundedness criteria in retarded Volterra integro-differential equations, 25, (2017), 363- 368. https://doi.org/10.1016/j.joems.2017.05.001.

[17] C. Tunc., New Stability and boundedness results to Volterra integro-differential equations with delay, Journal of the Egyptian Mathematical Society, 24(2016): 210-213. https://doi.org/10.1016/j.joems.2015.08.001.

[18] C. Tunc, Qualitative properties in nonlinear Volterra integro- differential equation with delay, J. Taibah Univ. Sci. (2016), https://doi.org/10.1016/j.jtusci.2015.12.009.

[19] J. Vanualailai, and S. Nakagiri,., Stability of a System of Volterra integro-differential equations. J. Math. Anal. Appl., Vol. 281(2003): 602-619. https://doi.org/10.1016/S0022247X(03)00171-9.

[20] A. M. Wazwaz, Linear and nonlinear integral equations, methods and applications, Higher Education Press, Beijing, Springer, Heidelberg, 2011. https://doi.org/10.1007/978-3-642-21449-3. 